\title{
Abnormal Grain Growth Mechanism in the Twin-Roller Cast Al-Fe-Si Alloy in the Annealing Process
}

\author{
Y. Z. Zhu $\mathbb{D}^{1}{ }^{1}$ H. Peng, ${ }^{1}$ H. Huang $\mathbb{D}{ }^{2}$ and J. C. Li $^{1}$ \\ ${ }^{1}$ Department of Material Engineering, North China University of Technology, Beijing 100144, China \\ ${ }^{2}$ College of Architecture and Civil Engineering, Beijing University of Technology, Beijing 100124, China
}

Correspondence should be addressed to Y.Z.Zhu; tozyz1@163.com

Received 12 April 2020; Revised 7 May 2020; Accepted 20 May 2020; Published 15 June 2020

Academic Editor: Stanislaw Dymek

Copyright (c) 2020 Y. Z. Zhu et al. This is an open access article distributed under the Creative Commons Attribution License, which permits unrestricted use, distribution, and reproduction in any medium, provided the original work is properly cited.

\begin{abstract}
The twin-roller casting process is a novel technology used to produce aluminium and other alloys. In this study, the Al-Fe-Si alloy was twin-roller cast into a billet with a thickness of $7.0 \mathrm{~mm}$. This was followed by an annealing process at $480^{\circ} \mathrm{C}$ for 16 hours. Abnormal grains of more than $15 \mathrm{~mm}$ in the nominal diameter were found to have formed on the surface of the billet. Scanning electronic microscopy observation, energy diffraction spectrum, microarea X-ray diffraction, and electronic backscattered diffraction analysis were performed to study this abnormal grain growth. The results showed that abnormal grain nucleates in the region of $(100)<001>$ texture formed in the twin-roller casting process. The growth of abnormal grain was governed by the coalescence of the abnormal grain with its surrounding normal grains, with an average orientation angle of $47^{\circ}$ between both the types of grains. High-strain-concentrated regions on the surface layer of the billet were induced by the heterogeneously distributed Fe particles formed in the twin-roller casting process. The presence of these regions accelerates the abnormal grain growth in the following annealing process of the billet.
\end{abstract}

\section{Introduction}

The twin-roller casting (TRC) process is a novel technology that exhibits higher energy efficiency than the traditional continuous casting process [1]. Semisolidus metals are both solidified and deformed in the gap between two hollow rollers rotating in opposite directions. The rollers are cooled by the circulation of cool water in them. The sheets and foils of alloys such as aluminium and copper are produced using this technology to lower the energy consumption. The thickness of the billet produced by the TRC process is often less than $10 \mathrm{~mm}$. This results in a cooling rate in the solidification process that is much higher than in the traditional casting process [2]. Both a finer granular microstructure and a slighter macrosegregation of alloy elements can be achieved in the TRC billet [3]

Moreover, the special solidification characteristics of the TRC process result in not only the special distribution of secondary precipitates but also the special grain orientation distribution in the billet [4]. The fine secondary precipitates are resulted from the relatively high cooling rate in the billet in the solidification process. These precipitates grow slowly because the duration time of the billet at elevated temperatures is short in this solidification process. However, there is still presence of a temperature difference between the centre and edge surface of the TRC billet, which results in different particle sizes in different regions in the billet. The grain orientation distribution at the surface of the billets may result from both the heterogeneity of the thermal field and the shearing strain from the rollers during the solidification process. This implies that the TRC billet has special heterogeneously distributed secondary precipitates and a special orientation distribution. An annealing process is often necessary before rolling this kind of TRC billet with the heterogeneous microstructure. This raises the question as to how this kind of special microstructure evolved in the annealing process.

Those factors such as the deformation texture, volume fraction, and the size of the secondary precipitates in alloys have all been argued to affect grain growth in the annealing 
process [5-7]. Abnormal grains have even been observed in alloys such as aluminium and copper, which ultimately affects the quality of these products $[5,6,8-10]$. It is therefore important and necessary to understand the possible further evolution of the special microstructure formed in the TRC billet in the annealing process. The purpose of this article is to understand the effect on the grain growth of the special microstructure in TRC billet in the following annealing process.

1.1. Experimental Works. AA1235 aluminium alloys were twin-roll cast into a billet with a thickness of $7.0 \mathrm{~mm}$ at $684^{\circ} \mathrm{C}$ in the air. The casting speed was $1100 \mathrm{~mm} / \mathrm{min}$. The chemical compositions of the AA1235 aluminium alloy are listed in Table 1.

Following the twin-roller casting process, the billet was annealed at $480^{\circ} \mathrm{C}$ for 16 hours in a muffle furnace and then taken out of the furnace and cooled to room temperature in the air. Samples were sectioned along the central line parallel to the rolling direction with dimensions of $7 \mathrm{~mm} \times 10 \mathrm{~mm} \times 20 \mathrm{~mm}$.

The surface of the billet was washed in a $2 \% \mathrm{NaOH}$ solution and then etched with an alcohol solution with 5 wt. $\% \mathrm{HNO}_{3}$ to reveal the microstructure. The microstructure was then observed and analyzed using optical microscopy and field emission electronic microscopy Siron 200 (FEI, Amsterdam, Netherlands) attached with an EDS analyser. The microstrain in the grains at the billet surface was measured using the Micro-XRD diffraction D/Max Rapid IIR diffraction system, with $\mathrm{CuKa}$ radiation, and a power of $10 \mathrm{~kW}$. The method for measuring the microstress is as follows. At first, the lattice parameters at different regions were measured using the XRD system. The microstrain can be calculated according to the measured parameters using the software Jade 10. Then the microstress can be calculated using the following equation:

$$
S=E \times u,
$$

where $S$ denotes the microstress, $E$ represents the elastic modulus, and $u$ is the microstrain. The orientation angle between the grain and the matrix was measured by the EBSD detector attached to the SEM system.

\section{Results}

The surface of the TRC billet was carefully checked. No abnormally coarsened grains were observed. The microstructure of the TRC billet is shown in Figure 1(a). EDS analysis shows that the main precipitates in the TRC billet are iron enriched ones (Fe precipitates). The microstructure of the TRC billet surface after annealing is presented in Figure 1(b). These show that an abnormally coarsened grain formed on the surface of the annealed TRC billet. This was much larger than the surrounding grains with a size of $15 \mathrm{~mm}$. Clear boundaries could be observed between the abnormal grain and its surroundings, indicating that it was fully recrystallized. A trace of shearing deformation was still visible in the microstructure of the matrix surrounding the abnormal grain, implying that the matrix was composed of nonfully recrystallized grains, namely, normal grains. A difference in colour was observed between the abnormal grain and the surrounding normal grains, implying a high orientation angle between the two. Furthermore, the trace of a tip growth was visible in the microstructure along the rolling direction of the billet.

The microstrain across the abnormal grain was measured using the Micro-XRD diffraction D/Max Rapid IIR system. The results are presented in Figure 1(c). These show that the microstrain in the abnormal grain is only $0.065 \%$ in average. The microstrain at the outside of this grain increases to approximately $0.135 \%$, more than twice that inside the grain. The changes in the microstrain from the abnormal grain to the surrounding normal grain imply a difference in dislocation density between these two regions.

SEM and EBSD analysis were employed to understand the detailed microstructure and orientations in the abnormal grain and its adjacent normal grains. The results are presented in Figures 2 and 3. These show that the microstructure near the abnormal grain contains three layers (Figure 2(a)). The core layer is the abnormal grain, while the outer layer comprises the nonfully recrystallized grains. Notably, there is a transitional layer between the abnormal grain and the nonfully recrystallized grain layer. The boundary between the transitional layer and the outer layer is fairly smooth. By contrast, there are numerous "zigzags" at the boundaries between the transitional layer and the abnormal grain (core layer) (Figure 2(a)).

Spot detection was performed by the EBSD near the abnormal grain. Clear Kikuchi patterns can be observed only in spots 7,6,5, and 4 (Figure 2), implying there is a much higher residual microstrain in the outer layer than in the abnormal grain and the transitional layer. Even in these latter two layers, the calibration rate of the Kikuchi patterns decreases as the distance from the centre of the abnormal grain to the boundary between the transitional layer and the outer layer decreases (Figure 2). This indicates an increasing residual microstrain from the centre of the abnormal grain to the outer layer, which is coincident with the $\mathrm{X}$-ray results (Figure 1(c)).

Fe precipitates at grain boundaries may have important effects on abnormal grain growth. Chemical compositions were measured by EDS analysis across the abnormal grain (Figure 3(a)). The results (Figure 3(b)) show that the contents of $\mathrm{Fe}$ in the abnormal grain, the transitional grain, and the grain of the outer layer are similar, while there is a substantial increase in $\mathrm{Fe}$ content at the interfaces (grain boundaries) between the outer layer and the transitional layer and between the transitional layer and the abnormal grain. This indicates that the Fe precipitates on the grain boundaries are not completely dissolved in the annealing process.

The features of the Fe precipitates were further investigated using SEM. The results are presented in Figure 4. These show the presence of clear triangular grain boundaries between the normal grains in the outer layer. Fe precipitates are distributed continuously along these grain boundaries, indicating that these boundaries are stable. However, the 
TABle 1: Chemical composition of the TRC and HR sheets for foils (wt.\%).

\begin{tabular}{lccccc}
\hline Element & $\mathrm{Fe}$ & $\mathrm{Si}$ & $\mathrm{Zn}$ & $\mathrm{Mg}$ & $\mathrm{Ti}$ \\
\hline Content & 0.39 & 0.10 & 0.015 & 0.007 & 0.011 \\
\hline
\end{tabular}

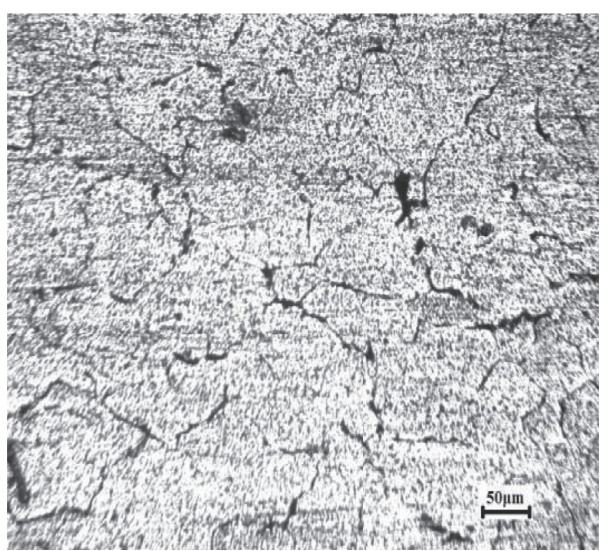

(a)

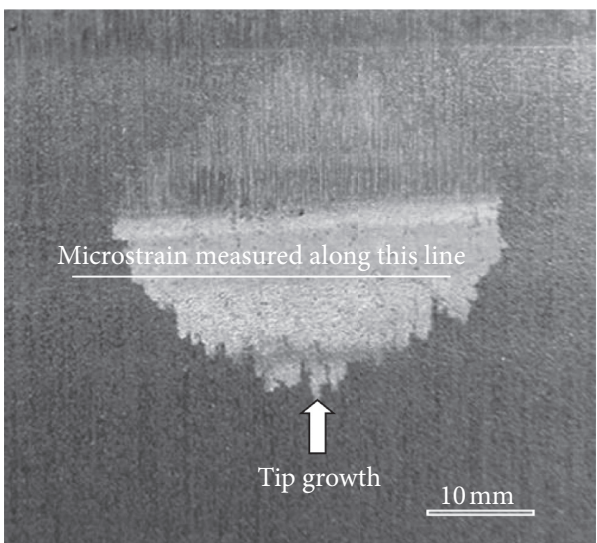

(b)

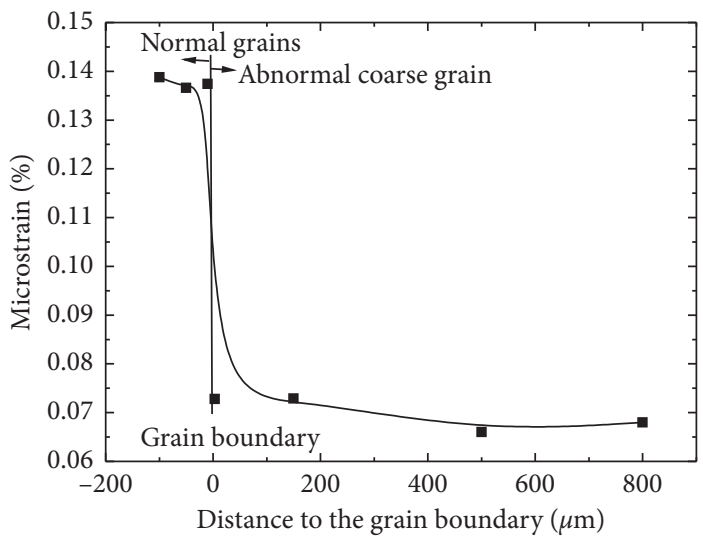

(c)

FiguRE 1: Macrographs of the abnormal grains in annealed twin-roller cast 1235 aluminium alloys. (a) The optical microstructure of the twin-roller cast alloy. (b) Morphology of the abnormal grain formed in the following annealing process. (c) Microstrain across the abnormal grain.

shape of the boundaries is irregular. Notably, the shape of the grain boundary of the abnormal grain is that of a smooth curve. This is occupied by discontinuously distributed Fe precipitates, implying that this type of grain boundary is unstable. The thickness of $\mathrm{Fe}$ precipitates at the grain boundary of the abnormal grain is much smaller than that at normal grain boundaries in the outer layer. The thick layer of the grain boundary with continuously distributed Fe precipitates will act to resist grain boundary (GB) migration in the annealing process. By contrast, the thin layer of the abnormal grain boundary with the discontinuously distributed Fe precipitates is susceptible to migrate, resulting in abnormal grain growth. The abnormal grain boundary also penetrates into the notch of the discontinuous grain boundary, implying a higher grain boundary migration rate at a location where there are fewer Fe precipitates (as shown in Figure 4(b)). Traces of the semidissolved Fe segregation cluster continue to be visible near the abnormal grain boundary (as shown in Figure 4(b)). The partially dissolved $\mathrm{Fe}$ precipitates result in the rapid migration of grain boundaries into the notch of the Fe precipitates "wall," giving rise to abnormal grain growth.

\section{Discussion}

3.1. Evolution of the Fe Precipitates in Annealing and Its Effect on Grain Growth. The original Fe precipitates formed in the twin-roll cast process are dissolved into the matrix of the alloy in the annealing process. These are usually about several microns in size. A large number of the precipitates are distributed at grain boundaries, which would usually resist grain boundary migration. Some of the precipitates are also distributed in clusters at the surface of the billet [7]. If these precipitates are dissolved, the grain will grow.

The microstructure of the abnormal grain boundary is illustrated in Figure 5. This shows that a large number of 


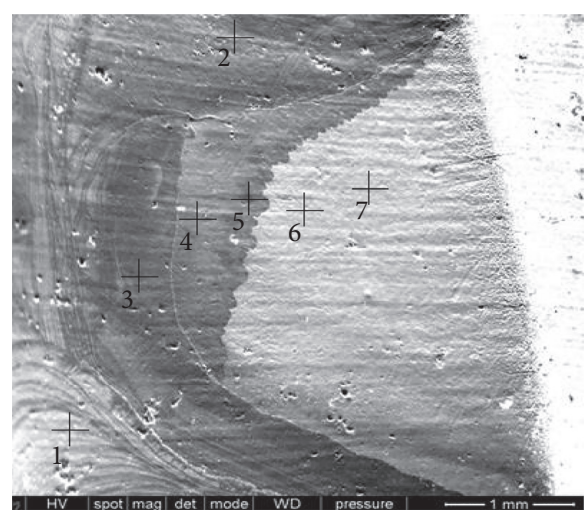

(a)

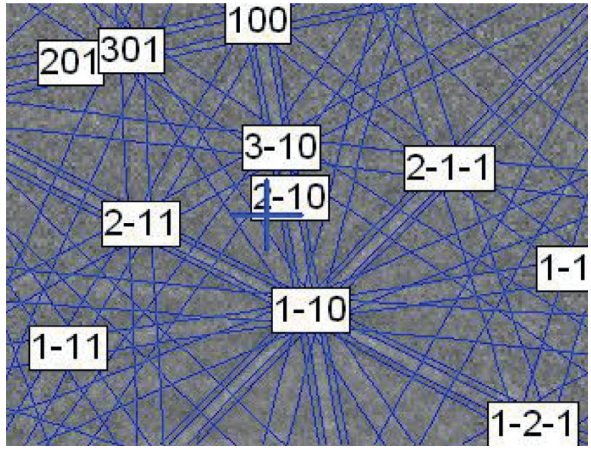

(c)

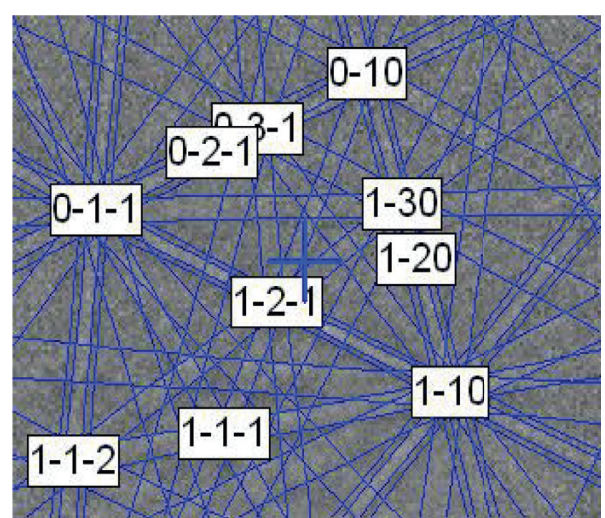

(b)

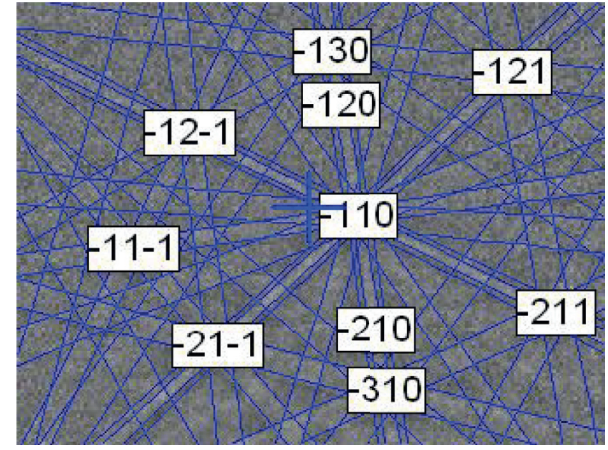

(d)

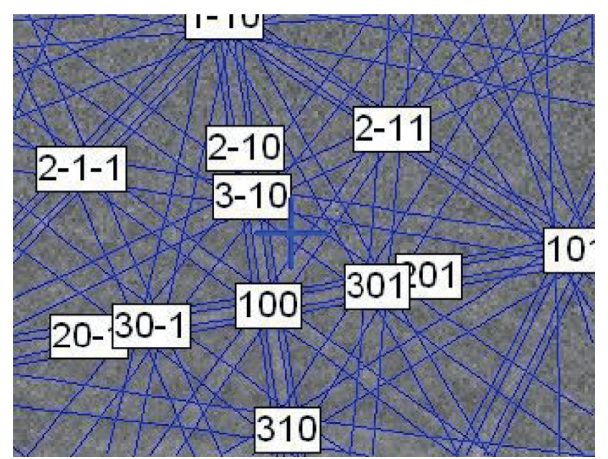

(e)

Figure 2: Transitional grains between abnormal grain and the matrix (orientation gradient and stress). (a) SEM micrograph of the abnormal grain (b-e) corresponding to the points $3,5,6$, and 7 in (a).

spherical Fe particles are distributed in clusters near the abnormal grain boundary (Figure 5(a)). The shape of the grain boundary section enriched by Fe precipitates implies that these precipitates originate from the partially dissolved original and large precipitates in the TRC billet. These Fe precipitates are distributed like a wall, which resists grain boundary migration. The abnormal grain boundary has not yet penetrated through this region, implying that the high density and large size of these particles is sufficient to prevent the migration of the abnormal grain boundary [11].

In another section of the abnormal grain boundary (shown in Figure 5(b)), the density of the Fe-enriched particle at or near the abnormal grain boundary is much lower than that shown in Figure 5(a). There is an obvious notch in the particle wall, where the section of the abnormal grain boundary has a curvature with a radius smaller than that of the adjacent grain boundary section, indicating rapid migration of the abnormal grain boundary in this direction. The centre of the notch (shown in Figure 5(b)) exhibits a low resistance to the migration of the abnormal grain boundary for a lower number of $\mathrm{Fe}$ precipitates. Furthermore, the dissolving particle wall adjacent to this notch provides a high density of solutes for this diffusion-dependent grain boundary migration process. The smaller the curvature radius of the grain boundary, the higher the growth rate of the grain has [12]. The abnormal grain boundary also has a bigger radius curvature when a high density of Fe particles is distributed at or near the abnormal grain boundary (as shown in Figure 5(c)), indicating that the resistance to grain boundary migration is enhanced by the high density of Fe particles. 


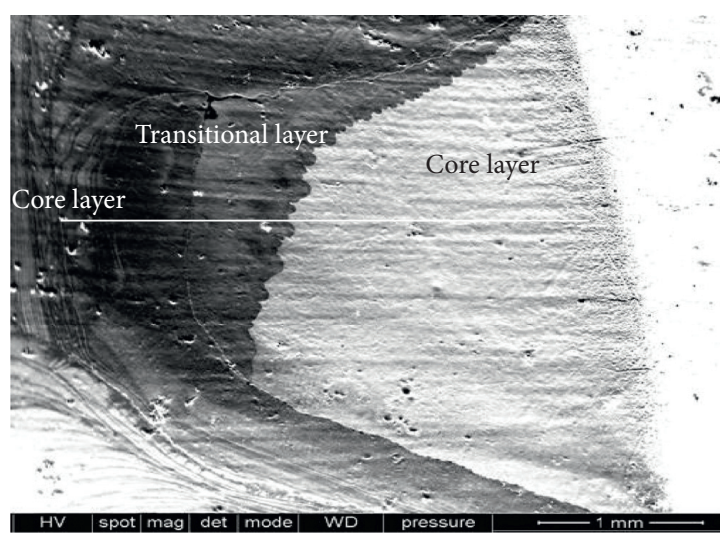

(a)

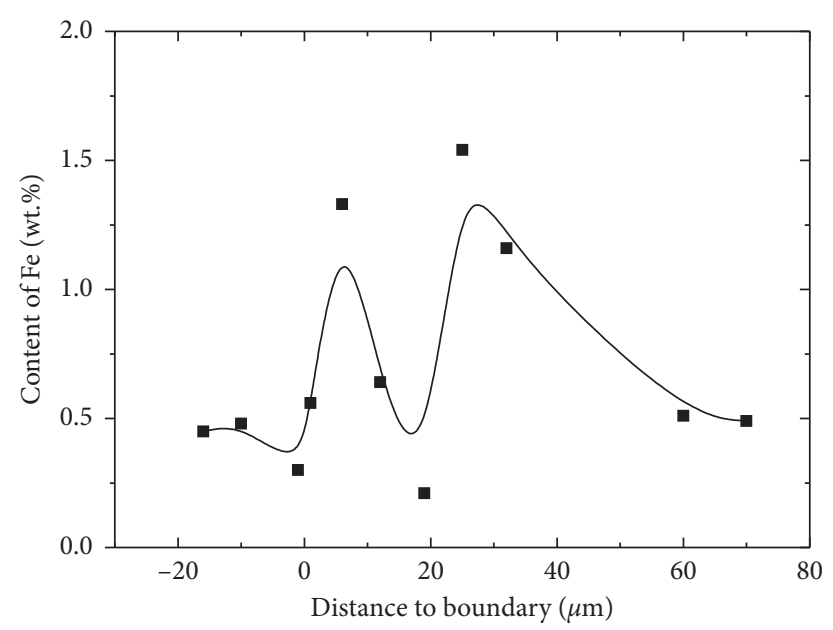

(b)

Figure 3: Hardness near the grain boundary of the abnormal grain. (a) Micrograph of the grain boundary. (b) Hardness across the grain boundary of the abnormal grain.

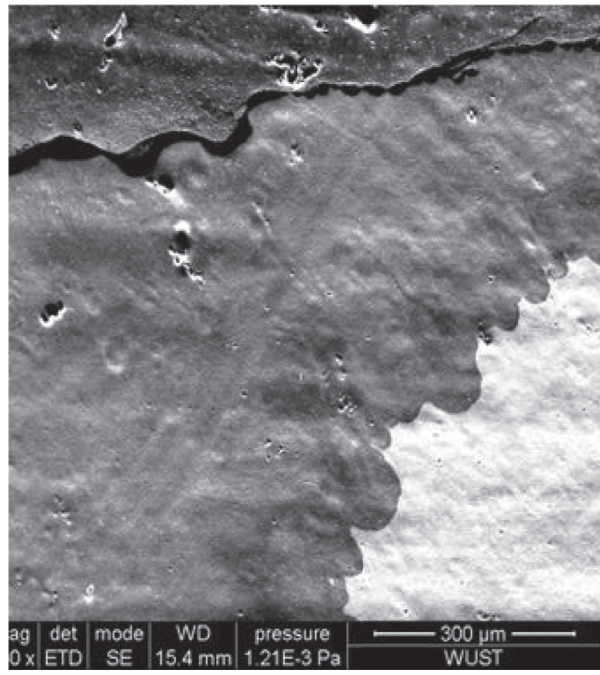

(a)

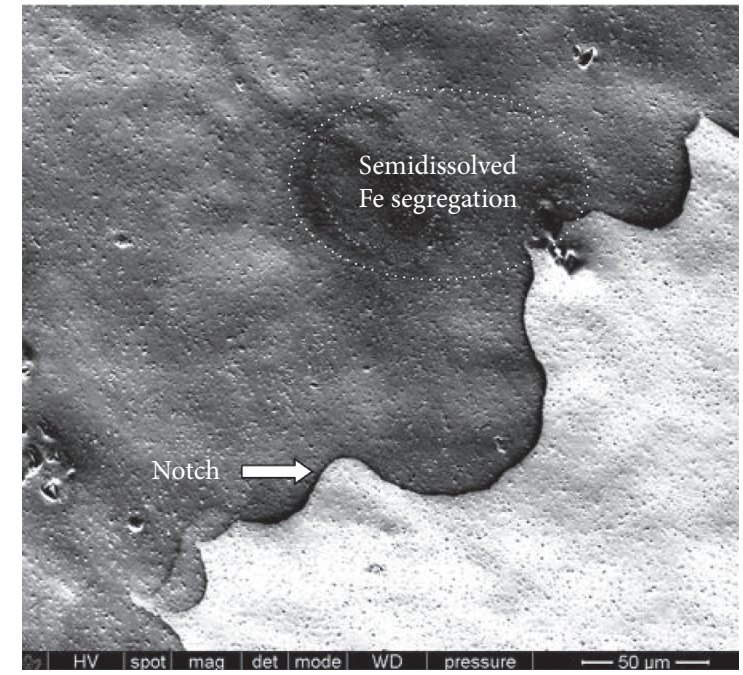

(b)

FIgURE 4: Fe precipitates at grain boundaries. (a) Three-layer structure of the abnormal grain boundary. (b) Partially dissolved particles at frontier of the grain boundary.

The results also show the abnormal grain boundary tends to penetrate the interface between the region with a high density of $\mathrm{Fe}$ particles and the region with a low density (Figure 5(d)). A high gradient of Fe content leads to a rapid diffusion of the element at the interface, causing dramatic growth in the abnormal grain at the transitional interface between the region with a high content of solutes and the region with a low content. The rate of grain growth of the alloy depends on the size of the secondary particles and its volume fraction [11].

If the particles are arranged in a wall, the growth of the abnormal grain can be resisted (Figure 6(a)). However, the resistance of a single isolated particle to grain growth is extremely limited (Figure 6(b)). Through the bugling mechanism, the abnormal grain easily penetrates its surrounding grains along the edge of Fe particles. This is because this kind of microlocation usually has a higher strain than other microlocations, which is caused by the strain concentration in the rolling process. The high gradient of the solid solutes at the edge of the Fe particles also provides sufficient conditions for rapid atomic diffusion at this location in the annealing process (Figure 6(c)). The curvature radius shown in Figure 6(c) is fairly small, which results in ultrahigh grain growth in the annealing process. TEM micrograph (Figure 6(d)) of the annealed alloy shows that the partially dissolved $\mathrm{Fe}$ precipitates cannot stop the coalescence of the adjacent grains.

AlFe and AlFeSi particles exhibit varying stability at the annealing temperature [13]. For instance, AlFe particles will dissolve earlier than AlFeSi particles, resulting in a more 


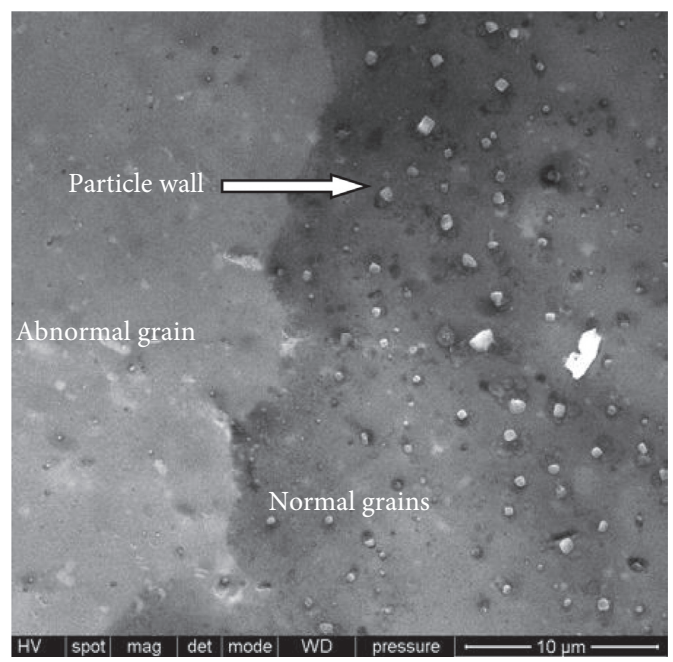

(a)

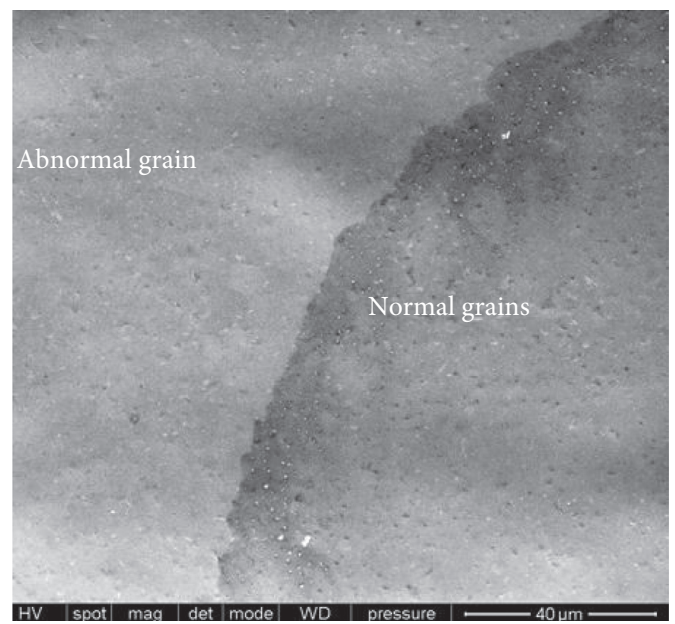

(c)

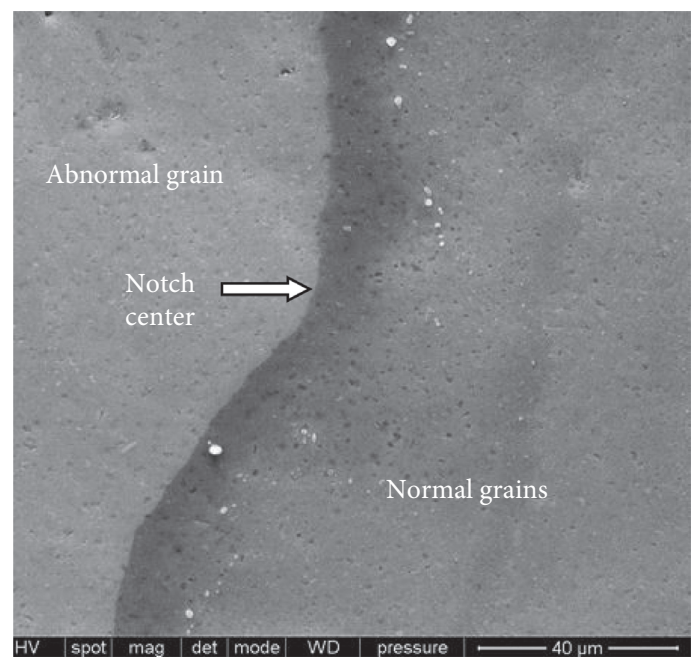

(b)

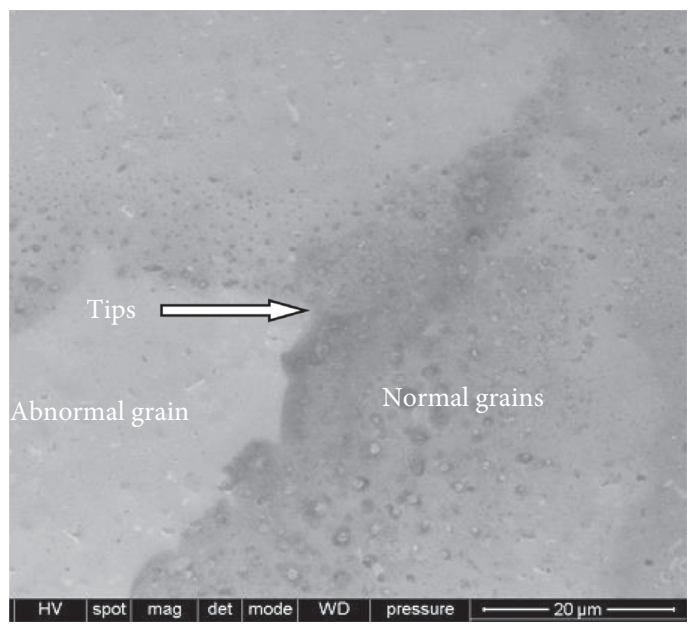

(d)

Figure 5: Precipitates at the grain boundaries of the abnormal grain. (a) The wall of particles to resist to GB migration. (b, c) GB bulging at the location with partially dissolved precipitates. (d) GB penetrating the location with high gradient of precipitates.

heterogeneous distribution of particles in the alloy. Thus, abnormal grain growth takes place. This phenomenon was also reported by Rios [14].

\subsection{Effect of the Original Fe Precipitates in the TRC Billet on} Grain Growth in the Subsequent Annealing Process. Because both solidification and deformation cooccur in the gap between two rotating rollers, the TRC billet usually possesses a microstructure different from that of the traditional cast billet. Clusters of precipitates can often be seen on the surface of the TRC billet [3]. If Fe segregation is not eliminated completely, stress concentration will be observed near this segregation. Figure 7 depicts the trace of a severe deformation near the Fe segregation, indicating a form of stress concentration in the "soft" matrix near the "hard" Fe segregation in the rolling process. The severe deformation at this region will provide high energy for grain growth. The heterogeneous distribution of $\mathrm{Fe}$ phase results in a heterogeneous distribution of strain in the alloy in the rolling process. This has been found to result in abnormal grain growth in the annealing process [15]. Thus, microlocations with a higher strain will lead to the formation of abnormal grains on the surface of the annealed alloy sheet due to rapid grain growth in the annealing process.

3.3. Orientation Evolution of the Abnormal Grain and Its Surroundings in the Annealing Process. EBSD analysis was performed to investigate the orientation between the abnormal grain and its adjacent normal grains. The results are presented in Figure 8. These show that the orientations within the abnormal grain are the same, implying that it is a single crystal. Moreover, most of the normal grains have low angle boundaries with their adjacent normal grains, indicating the existence of a texture in the normal grains surrounding the abnormal grain. For further confirmation of the specific texture around the abnormal grain, the pole figure (PF) and inverse pole figure (IPF) are presented in Figure 9. The PF shows a $<001>$ orientation in the RD, TD, 


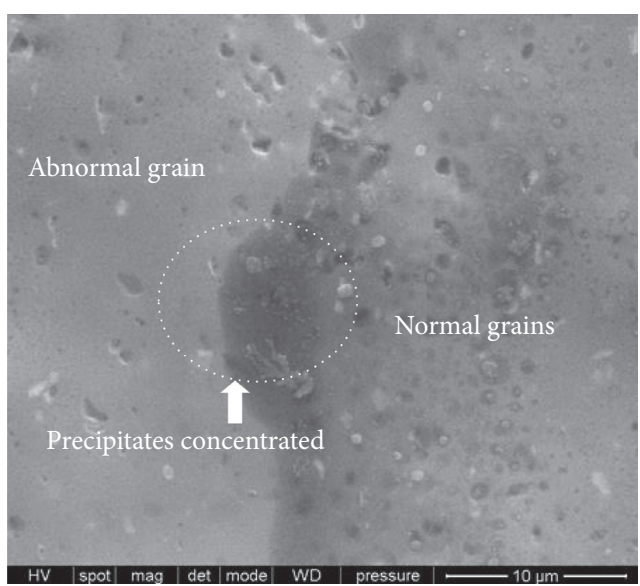

(a)

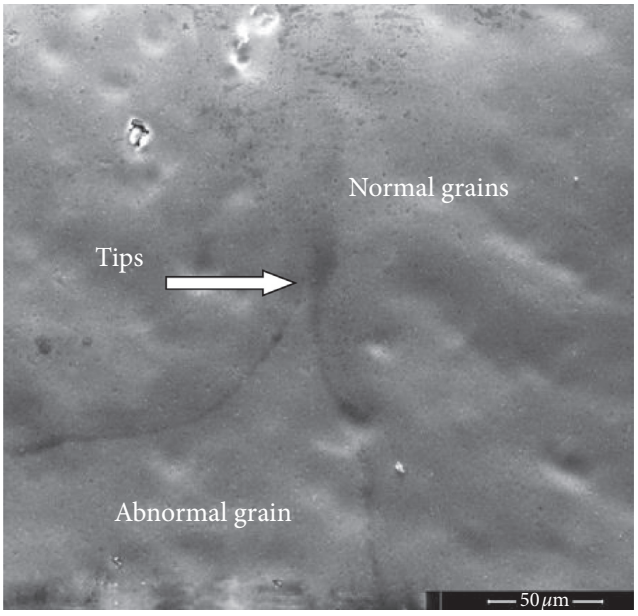

(c)

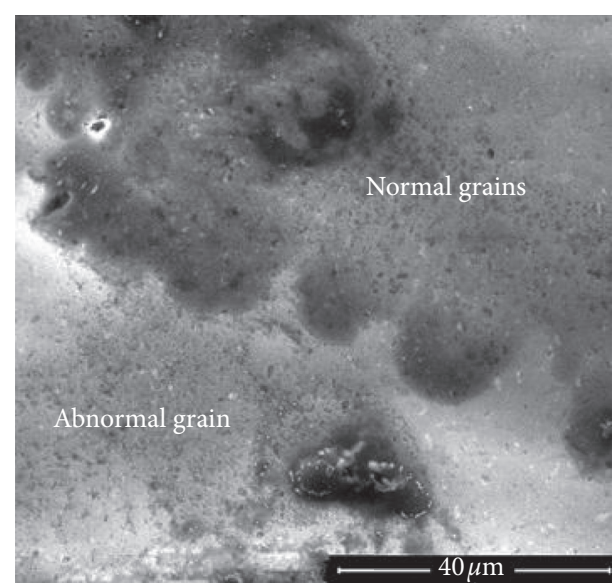

(b)

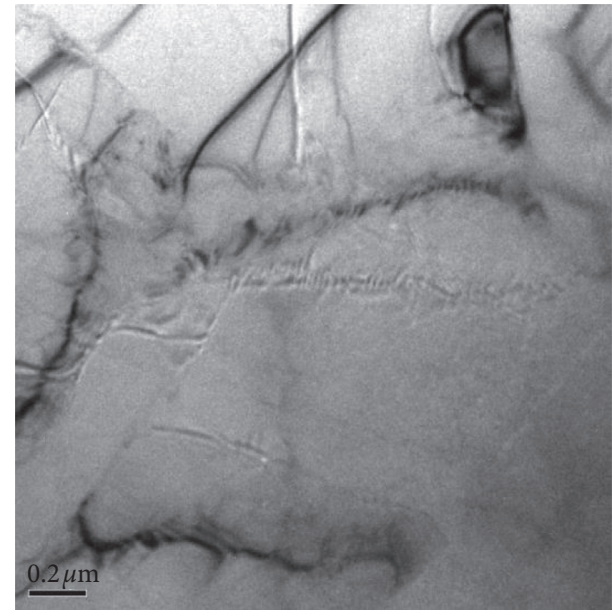

(d)

FIGURE 6: Growth penetrating into the semidissolved Fe-enriched coarse particles. (a) The dissolution of coarse precipitates leading to bugling of GB. (b) Zigzags at the location with partially dissolved original segregation. (c) Rapid bugling of GB into the location with high content Fe. (d) TEM micrograph of the annealed alloy.

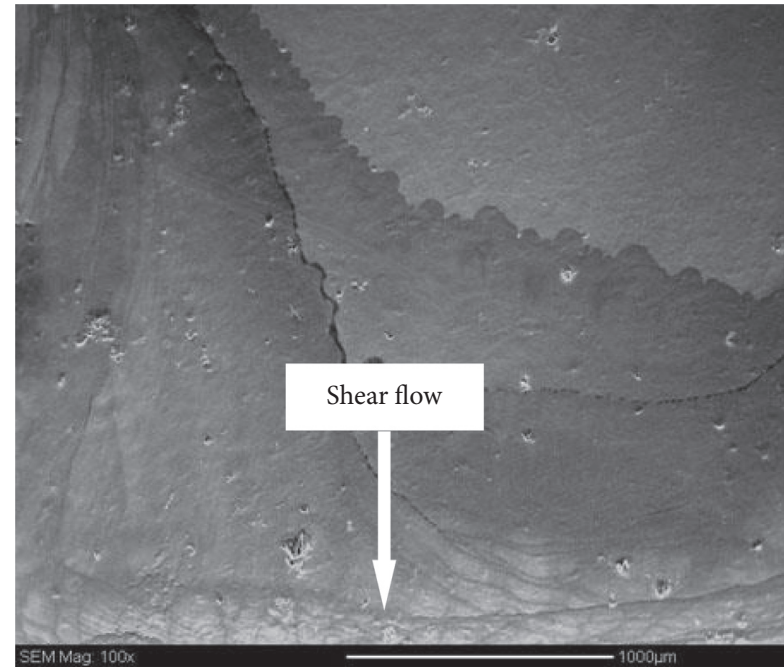

FIGURE 7: Obvious trace of shear flow of metals near nondissolved Fe-enriched precipitates wall. and ND directions. The IPF shows that it is almost a standard (100) IPF. This confirms that the texture adjacent to the abnormal grain is $(100)<001>$. This has been found to be a typical hot deformation texture in aluminium alloys after cold rolling [16]. The cubic texture has also been reported to result in abnormal grain growth [5]. The presence of strain in grains with this kind of texture suggests that this texture is formed or evolves from another texture in the twin-roll casting process. This is because the two rotating rollers contacting the billet cause strong friction and thus high strain on the surfaces of the billet. Consequently, a texture will be formed on the billet surfaces.

The orientation angle between the texture and the abnormal grain can be calculated using the Euler rotation method. First, a point (such as point 1) on the abnormal grain is analyzed using EBSD (as shown in Figure 10). The Euler angle of point 1 is $(122,14.6,4)$. The orientation angle between the abnormal grain and its surrounding texture can be calculated as shown in Figure 11. This was found to be $47^{\circ}$. 


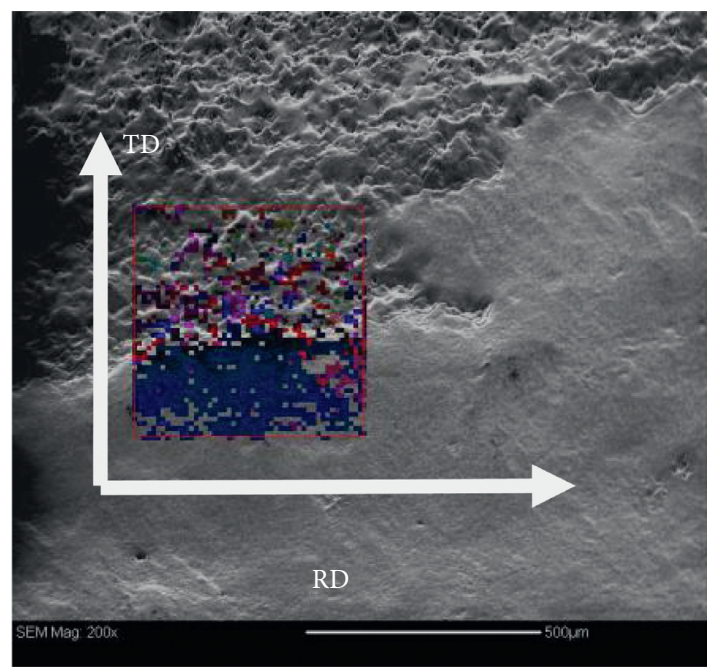

(a)

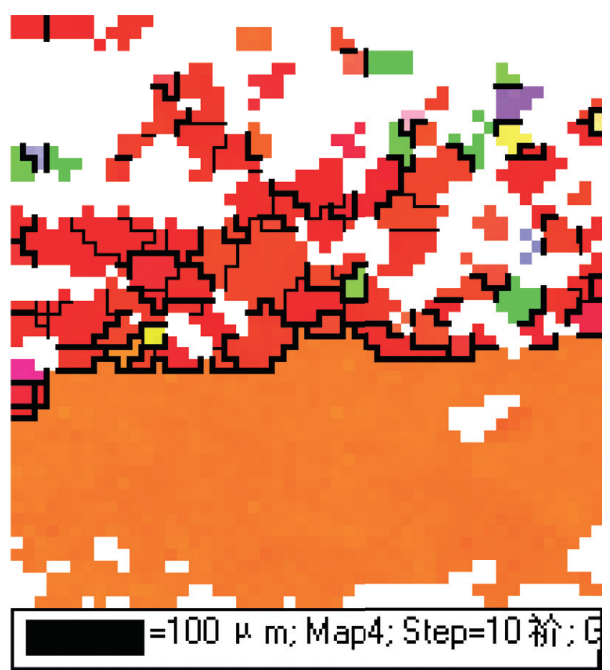

(b)

FIGURE 8: EBSD analyzing of the abnormal grain. (a) Location for EBSD analysis. (b) The results of the EBSD analysis.

$\mathrm{RD}$

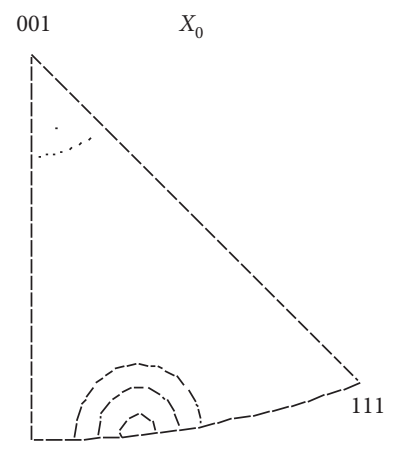

101
TD

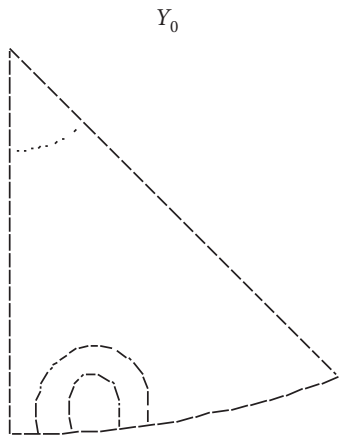

(a)

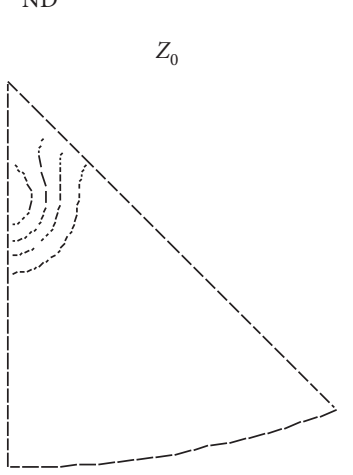

\begin{tabular}{|l|}
$\begin{array}{l}\text { Inverse pole figures } \\
\text { (folded) }\end{array}$ \\
\hline [Project4.cpr] \\
Aluminium $(\mathrm{m} 3 \mathrm{~m})$ \\
Complete dataset \\
1678 data points \\
Equal area projection \\
Upper hemispheres \\
\hline \hline Half width: $10^{\circ}$ \\
Cluster size: $5^{\circ}$ \\
Exp. densities (mud): \\
Min $=0.00$, Max $=10.59$ \\
2
\end{tabular}

$\mathrm{RD}$

TD
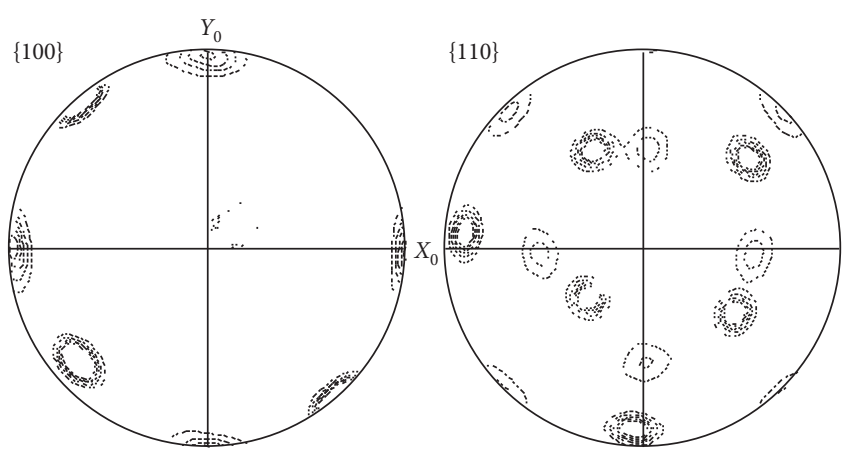

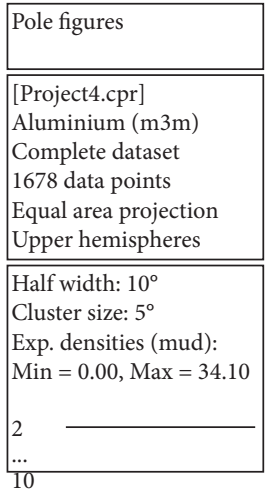

(b)

Figure 9: Pole figure and inverse pole figure near the abnormal grain boundary: (a) IPF; (b) PF.

The grain boundaries can usually be generalised into three categories: (1) $\Sigma 1$ grain boundary when the grain boundary orientation angle between adjacent grains is from $2^{\circ}$ to $15^{\circ},(2)$ coincidence lattice grain boundary when $3 \leq \Sigma \leq 29$, and (3) random grain boundary when $\Sigma>29$. The sigma value $\Sigma$ between the adjacent normal grains and the abnormal grain was then calculated. The results are presented in Figure 12. These show that CSL grain boundaries between the adjacent 


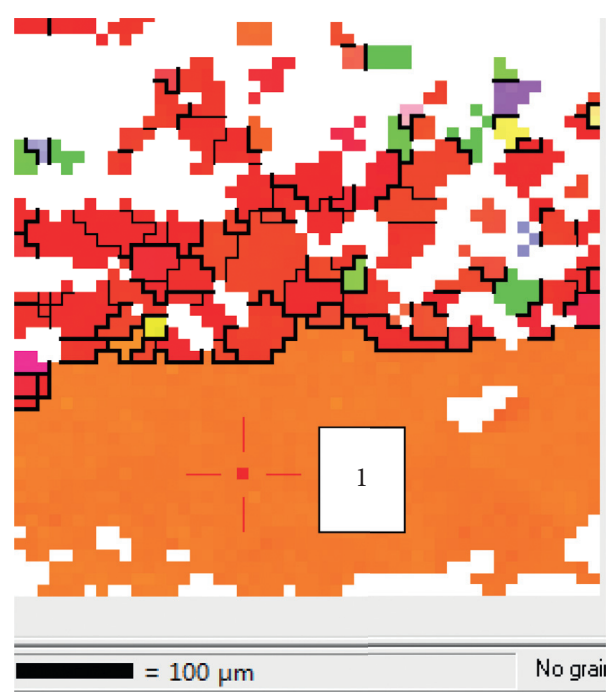

Figure 10: The point for Euler angle analysis.

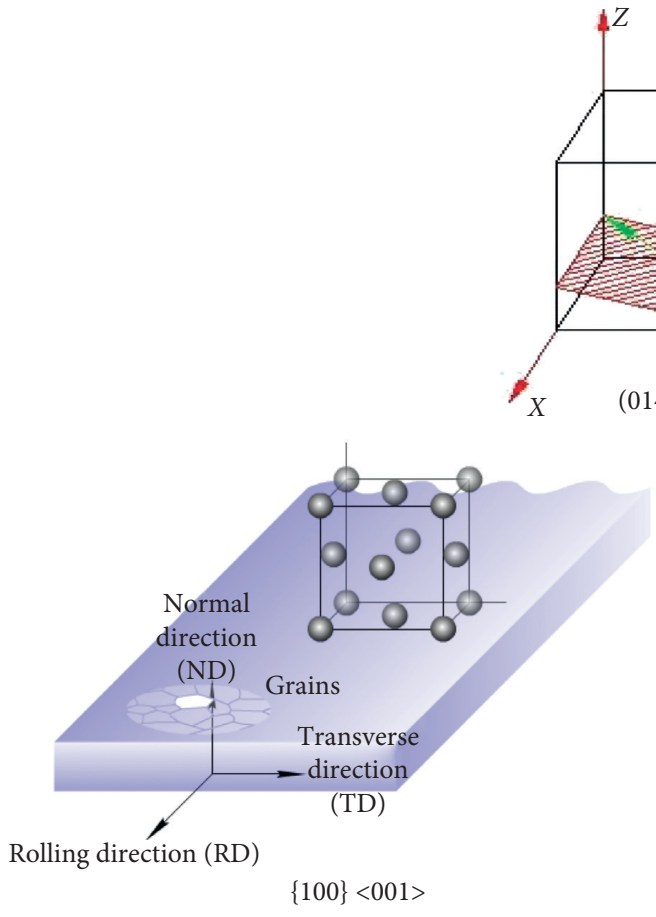

(a)

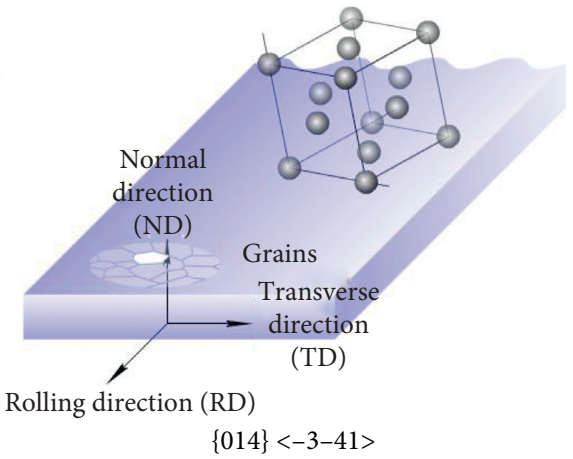

(b)

FIGURE 11: The orientation of the abnormal grain in the billet. (a) The normal grain at the surface of the billet. (b) The abnormal grain.

normal grains are extremely rare. Moreover, there are no CSL grain boundaries between the abnormal grain and the adjacent normal grains; instead, only random grain boundaries can be seen. The orientation angle between the abnormal grain and its surrounding normal grains is greater than $45^{\circ}$ and is thus a typical high angle grain boundary. A high angle grain boundary results in high energy. Therefore, the abnormal grain will frequently grow in the form of rotation coalescence recrystallization by lowering the free energy of the system.

3.4. Growth Mechanism of the Abnormal Grain. In general, heterogeneity is evident in the TRC plate; in particular, the secondary phase containing $\mathrm{Fe}$ is usually distributed in clusters near the surface of the TRC billet. These clusters are not easily broken in the rolling process. The rolling process only results in strain concentration in the matrix near the edge of the clusters of Fe precipitates (see Figure 7). Thus, high energy storage is present in this region. At the same time, in subsequent annealing processes at elevated temperatures such as $480^{\circ} \mathrm{C}$, the clusters of Fe precipitates are partially dissolved (see Figures 4-6). This causes a high gradient of $\mathrm{Fe}$ in different locations in the matrix, which is due to the low diffusion rate of the element in the aluminium matrix. For example, there should be a low content of Fe rather than a high strain concentration in the matrix near the 


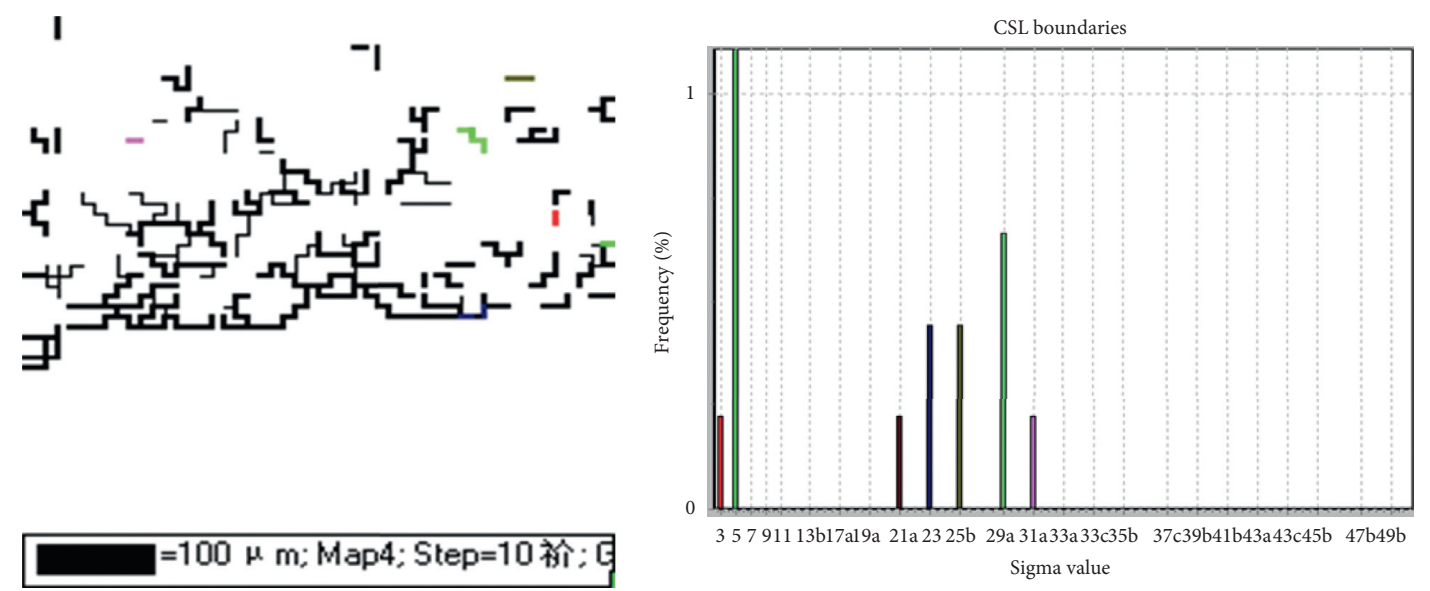

(a)

(b)

Figure 12: The CSL grain boundary of the abnormal grain and its surroundings.

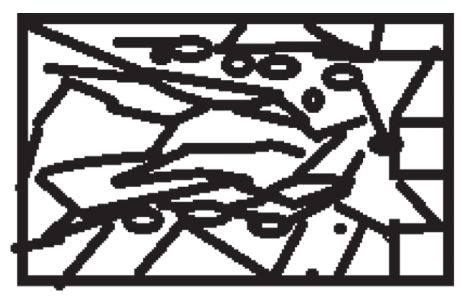

Twin-roll casting

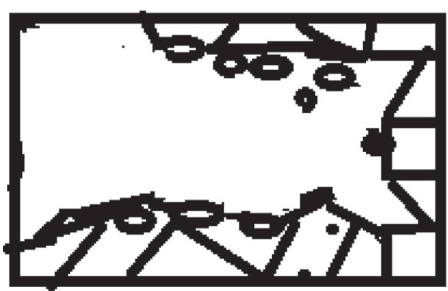

Abnormal grain growth by

GB bugling

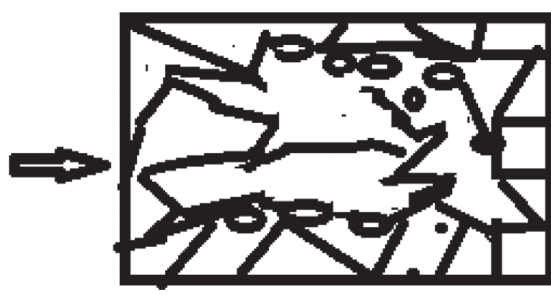

Nucleation at strain concentration sites
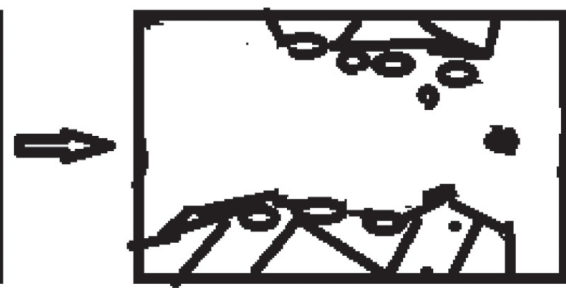

Abnormal grain growth by subgrain coalescence and rotation of high-angle GB

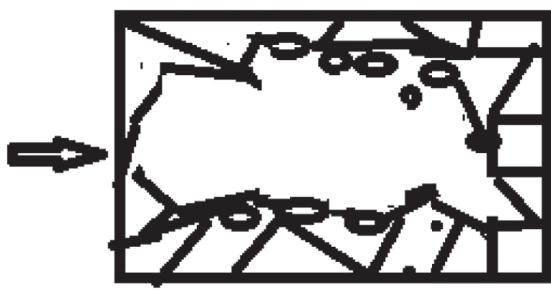

Abnormal grain formed

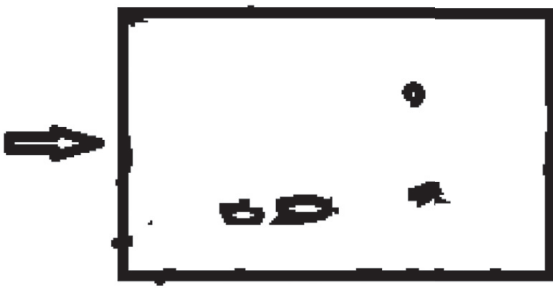

Frequent growth of grain

Figure 13: The mechanism for grain growth assisted by particle dissolution.

boundary with the precipitate clusters, which is where nucleation occurs in the annealing process. These nucleuses can grow very rapidly for the presence of high energy storage and low content of $\mathrm{Fe}$ at the boundaries of these kinds of regions. Moreover, the texture formed on the surface of the billet implies that these grains or subgrains are easy to grow according to the mechanism of subgrain coalescence.

The entire abnormal grain growth mechanism in the TRC billet during the annealing process is presented in Figure 13. The process is as follows: first, heterogeneously distributed precipitates and shearing strain coexist in some regions. At the same time, texture forms, assisted by shearing deformation (friction). Nuclei then form in regions with a lower density of precipitates. The adjacent nuclei have a similar orientation for the presence of texture. Reheating will dissolve or partly dissolve the precipitates between the adjacent nuclei. These nucleuses coalesce rapidly in the way of rotation. Thus, grains form. This is followed by the formation of high angle grain boundaries between these newly formed grains and surrounding grains with a high density of precipitates. When reheating continues, the newly formed grain will rapidly migrate to its grain boundary side with lower density precipitates and coalesce with an adjacent grain that has a similar orientation. This mechanism can be termed a friction-assisted grain growth mechanism.

This special abnormal grain growth mechanism possibly paves a new method for scientists to produce big single crystal. It also implies that the alloy can keep a relatively high strength because the grains would not grow rapidly if the annealing temperature is not high enough to dissolve or partially dissolve the Fe precipitates. 


\section{Conclusions}

An abnormal grain was found on the surface of the annealed twin roll cast billet. SEM, EBSD, and micro-X-ray diffraction were employed to analyse the mechanism for the growth of the abnormal grain. The following conclusions can be drawn:

(1) The partial dissolution of Fe precipitates in the billet results in a high gradient of Fe in the matrix, leading to rapid and abnormal growth of grains in locations with a lower content of Fe.

(2) There is a $(100)<001>$ texture on the surface of the billet. This facilitates the rapid growth of the surface grains in the annealing process.

(3) The boundaries between the abnormal grain and the adjacent grain are high angle boundaries. The orientation angle is $47^{\circ}$. To lower the free energy of the whole system, the abnormal grain will grow up by the coalescence of the adjacent grains during the annealing process by means of the rotation recrystallization mechanism.

The abnormal grain can possibly be eliminated by changing the texture formed in the twin-roller casting process if the parameters of the twin-roller casting are properly set up. A two-stage annealing may be a way to avoid the abnormal grain growth. The stress formed in the twinroller casting process can be removed at a relatively lower temperature; then the Fe precipitates would be dissolved at a higher temperature.

\section{Data Availability}

The data used to support the findings of this research are available from the corresponding author upon request.

\section{Conflicts of Interest}

The authors declare that they have no conflicts of interest.

\section{Acknowledgments}

The authors appreciate the support they have been given from the National Key R\&D Program of China (no. 2017YFE0117400) and the National Natural Science Foundation of China (no. 51675007).

\section{References}

[1] P. R. Beeley, "Some advances in the casting of metals," Materials \& Design, vol. 3, no. 6, pp. 632-637, 1982.

[2] E. Lavernia, G. Rai, and N. J. Grant, "Rapid solidification processing of 7XXX aluminium alloys: a review," Materials Science and Engineering, vol. 79, no. 2, pp. 211-221, 1986.

[3] Y. Z. Zhu, R. Y. Huang, Z. Zhu, and Z. D. Xiang, "Comparative study on effects of microstructures of hot rolled and twin roll casting 1235 aluminium alloy on surface quality of aluminium foils produced," Materials Science and Technology, vol. 27, no. 4, pp. 761-766, 2011.
[4] M. Slámová, M. Karlk, F. Robaut, P. Sláma, and M. Véron, "Differences in microstructure and texture of $\mathrm{Al}-\mathrm{Mg}$ sheets produced by twin-roll continuous casting and by direct-chill casting," Materials Characterization, vol. 49, no. 3, pp. 231-240, 2002.

[5] H.-C. Kim, C.-G. Kang, M.-Y. Huh, and O. Engler, "Effect of primary recrystallization texture on abnormal grain growth in an aluminum alloy," Scripta Materialia, vol. 57, no. 4, pp. 325-327, 2007.

[6] J. B. Koo and D. Y. Yoon, "Abnormal grain growth in bulk $\mathrm{Cu}$-the dependence on initial grain size and annealing temperature," Metallurgical and Materials Transactions A, vol. 32, no. 8, pp. 1911-1926, 2001.

[7] B. Forbord, B. Andersson, F. Ingvaldsen, O. Austevik, J. A. Horst, and I. Skauvik, "The formation of surface segregates during twin roll casting of aluminium alloys," $M a$ terials Science and Engineering: A, vol. 415, no. 1-2, pp. 12-20, 2006.

[8] H. Wei, J. Q. Tang, and D. Y. Xu, "Effect of abnormal grain growth on thermoelectric properties of hot-presses $\mathrm{Bi}_{0.5} \mathrm{Sb}_{1.5} \mathrm{Te}_{3}$ alloys," Journal of Alloys and Compounds, vol. 817 , pp. 1-8, 2020.

[9] S. Birosca, A. Nadoum, D. Hawezy, F. Robinson, and W. Kockelmann, "Mechanistic approach of goss abnormal grain growth in electrical steel: theory and argument," Acta Materialia, vol. 185, pp. 370-381, 2020.

[10] P. Sonnweber-Ribic, P. A. Gruber, G. Dehm, H. P. Strunk, and E. Arzt, "Kinetics and driving forces of abnormal grain growth in thin Cu films," Acta Materialia, vol. 60, no. 5, pp. 23972406, 2012.

[11] T. Gladman, "On the theory of the effect of precipitate particles on grain growth in metals," Proceedings of the Royal Society A, vol. 294, no. 1438, pp. 298-309, 1966.

[12] A. M. Deus, M. A. Fortes, P. J. Ferreira, and J. B. V. Sande, "A general approach to grain growth driven by energy density differences," Acta Materialia, vol. 50, no. 13, pp. 3317-3330, 2002.

[13] S. Nagy, Z. Homonnay, A. Vértes, and L. Murgás, "Mössbauer investigation of iron in aluminium-II. Al-Fe-Si samples," Acta Metallurgica, vol. 35, no. 3, pp. 741-746, 1987.

[14] P. R. Rios, "Abnormal grain growth development from uniform grain size distributions in the presence of stable particles," Scripta Materialia, vol. 39, no. 12, pp. 1725-1730, 1998.

[15] J. M. Feppon and W. B. Hutchinson, "On the growth of grains," Acta Materialia, vol. 50, no. 13, pp. 3293-3300, 2002.

[16] P. I. N. G. Liu, X-Ray Powder Diffraction for Materials Scientists: Principles and Practices, Sandvik Materials Technology, Sandviken, Sweden, 2012. 